\title{
Demystifying the NIH Grant Application Process: The Rest of the Story
}

\author{
Ronnie D. Horner, PhD \\ Department of Public Health Sciences, Institute for the Study of Health, University of Cincinnati Academic Health Center, Cincinnati, OH, USA.
}

$\mathrm{J}$ Gen Intern Med 22(11):1628-9

DOI: $10.1007 / \mathrm{s} 11606-007-0377-\mathrm{Z}$

(c) Society of General Internal Medicine 2007

I $\mathrm{n}$ this issue of the Journal, Dr. Berg and colleagues present their views on the NIH grant application review process in their article entitled, "Demystifying the NIH Grant Application Process". ${ }^{1}$ Having served at the NIH for a couple of years, I can attest that they have summarized the process with some accuracy and overall offer good advice and counsel. However, they have missed a few of the nuances to the process that I believe represent pieces of knowledge that are important for the individual who aspires to a career as an independent clinical investigator. Now here is the rest of the story.

First and foremost, one should keep in mind that each institute or center (referred to as IC by those at NIH) has its own culture or approach to the business of funding good science. So, if you are familiar with the modus operandi of 1 IC, you only know 1 IC.

Second, the NIH has 3 general mechanisms for carrying out its mission to improve the nation's health. Berg et al. discuss the first of these, which is the grant award where the investigator specifies what is to be achieved and how, and the $\mathrm{NIH}$ is essentially a patron, providing the funds (to those applications judged scientifically meritorious). ${ }^{1}$ Then, there are contracts under which the NIH specifies what is to be achieved and how, and investigators "bid" on the project. Finally, there is the lesser-known cooperative agreement where an IC is a partner with the investigator in designing and conducting a project. Cooperative agreements-funded under the U mechanism-tend to develop out of conversations between investigators and IC program officials and involve projects with aims for which an IC has a vested interest in seeing achieved. These are not entered into readily and, although a partnership, the project must still pass rigorous peer review to ensure its scientific merit. Still, a cooperative agreement is worth exploring for projects that are highly aligned with an IC's mission and with potential to significantly improve the nation's health.

It is also worth knowing that program announcements (PA's) can be of 3 types: PA, PAR, and PAS. Berg et al. discuss the PA. ${ }^{1}$ The PAR involves scientific review within the soliciting IC versus review by one of the integrated review groups (IRGs) at the Center for Scientific Review. Some believe these "in-house" reviews are more advantageous to the investigator. From my

Published online September 25, 2007 experience, whereas there may be fewer applications considered, the review is no less rigorous. The review panel, though, is comprised of those with considerable expertise in the area of the PAR so they understand the science very well. Whether this is an advantage is hard to say. The PAS has set aside funds, that is, funds dedicated to support scientifically meritorious applications solicited by the PAS. This arrangement clearly is advantageous to the applicant.

Review, whether of grant applications or manuscripts, is an inherently conservative process. I have served on review panels and, as a NIH program officer, watched review panels in action. The young investigator who has an anemic record of funded grants and published articles is at a distinct disadvantage. The NIH is well aware of the unequal playing field faced by the new investigator, and has tried to correct it. New investigator status is indicated on the application form and scientific review administrators (SRAs) - the individuals legally responsible for an impartial review process-remind panels when it is noted that an investigator is new that the application needs to be reviewed with this in mind. In my personal experience, I have rarely seen the new investigator accorded much leeway: the application is typically judged to the same level of scientific rigor accorded established investigators. Be that as it may, the new investigator can enhance their likelihood of success by good grantsmanship.

To paraphrase Louis Pasteur, "NIH favors only the prepared grant application”. By prepared, I refer to more than an application that conforms to the guidelines, although that is essential if you want your grant application reviewed. I also mean a narrative in which the objectives flow logically from the current gaps in knowledge and the study design and methodology are appropriate to answering the objectives. This is what is known as good grantsmanship and are the typical points of trouble for the novice grant writer. "Unfocused" grant applications invariably fail to propose methods that are appropriate to answering the objectives or fail to adequately describe the methods. It is not wasted effort to describe the rationale for selecting among available alternative approaches.

I also want to emphasize several other components of good grantsmanship. First, the NIH is interested in topics that have high clinical relevance. Applications where the clinical relevance is obvious and clearly delineated do better in review. Second, projects that are concise do better in review. If the summary statement describes your proposal as "ambitious', you have bitten off more than you can proverbially chew, at least as adjudicated by the reviewers. Third, applications with pilot data that provide direct support for the hypotheses and objectives do better. Of the last 3 points, this latter one is most important and should never be neglected. Because review is a 
conservative process, supporting evidence can help convince the reviewers that the project is meritorious.

Because of the odds against them, the novice clinical investigator must be persistent in seeking funding. However, the applicant should not write the grant application with the view that "No one ever gets funded the first time" or "I'll benefit from review". Write it to succeed the first time. As noted, poor grantsmanship is often the reason for failure. Again, the common errors are trying to do too much, failure to explicitly and clearly link the study objectives to the methodological approach, and failure to provide sufficient detail on the methods.

If your application fails to pass scientific review, a careful read of the review will generally inform you as to how to amend the application. The key operative is careful. Summary statements are often difficult to decipher. For example, poorly written grants may receive relatively few comments as there are so many flaws that it is not worth the reviewers' time and effort to identify them all. The ability to read between the lines is a skill that will assist the novice grant writer in achieving success on subsequent attempts.

In the Art of War, it states that if you know yourself and know your enemy, you will invariably be victorious. ${ }^{2}$ It is worth keeping in mind the purpose of $\mathrm{NIH}$ and our purpose as clinical investigators. The fundamental raison d'etre for NIH is to improve the nation's health. Whereas it may seem that there is a bias at NIH toward studies that address the mechanism of disease, the NIH ROADMAP initiative is a sincere attempt to rebalance funding priorities to give higher priority to projects that are not mechanistic in focus but seek to solve questions facing the clinician in everyday practice. It is a healthy trend. For the clinical investigator, the motivation should be to engage in studies that have high probability of yielding knowledge that will have a more direct and immediate impact on the individual's health through improvement in health behaviors and health care practices. There are many reasons a clinician becomes a clinical investigator; I hope the noble goal of improving health and health care would be the foremost impetus for embarking on this road.

Corresponding Author: Ronnie D. Horner, PhD; Department of Public Health Sciences, Institute for the Study of Health, University of Cincinnati Academic Health Center, P.O. Box 670840, Cincinnati OH 45267, USA (e-mail: Ronnie.Horner@uc.edu).

\section{REFERENCES}

1. Berg KM, Gill TM, Brown AF, Zerzan J, Elmore JG, Wilson IB. Demystifying the NIH grant application process. J Gen Intern Med. 2007 ; in press.

2. Griffith S, translator. Sun Tzu The Art of War. New York: Oxford University Press; 1963. 